\title{
OPEN Dispensable roles of Gsdmd and Ripk3 in sustaining IL-1ß production and chronic inflammation in Th17-mediated autoimmune arthritis
}

\author{
Yusuke Takeuchi ${ }^{1,3}$, Daiya Ohara ${ }^{1}$, Hitomi Watanabe ${ }^{1}$, Noriko Sakaguchi' ${ }^{2}$, \\ Shimon Sakaguchi ${ }^{2}$, Gen Kondoh ${ }^{1}$, Akio Morinobu ${ }^{3}$, Tsuneyo Mimori ${ }^{3,4}$ \& Keiji Hirota ${ }^{1 凶}$
}

Programmed necrosis, such as necroptosis and pyroptosis, is a highly pro-inflammatory cellular event that is associated with chronic inflammation. Although there are various triggers of pyroptosis and necroptosis in autoimmune tissue inflammation and subsequent lytic forms of cell death release abundant inflammatory mediators, including damage-associated molecular patterns and IL-1 $\beta$, capable of amplifying autoimmune Th17 effector functions, it remains largely unclear whether the programs play a crucial role in the pathogenesis of autoimmune arthritis. We herein report that Gasdermin D (Gsdmd) and receptor interacting serine/threonine kinase 3 (Ripk3)-key molecules of pyroptosis and necroptosis, respectively-are upregulated in inflamed synovial tissues, but dispensable for IL-1 $\beta$ production and the development of IL-17-producing Thelper (Th17) cell-

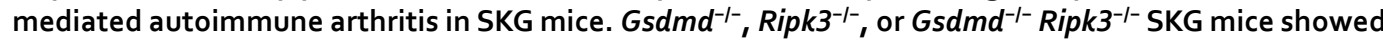
severe arthritis with expansion of arthritogenic Th17 cells in the draining LNs and inflamed joints, which was comparable to that in wild-type SKG mice. Despite the marked reduction of IL-1 $\beta$ secretion from $\mathrm{Gsdmd}^{-1-}$ or Ripk3 ${ }^{-1-}$ bone marrow-derived DCs by canonical stimuli, IL-1 $\beta$ levels in the inflamed synovium were not affected in the absence of Gsdmd or Ripk3. Our results revealed that T cellmediated autoimmune arthritis proceeds independently of the pyroptosis and necroptosis pathways.

Programmed necrosis associated with tissue inflammation is a newly reported concept of a lytic form of cell death that has emerged over the past few decades ${ }^{1}$. Pyroptosis and necroptosis, two distinct forms of programmed necrosis, not only play a crucial role in the host defense against infectious pathogens ${ }^{1-3}$, but have also been highlighted in various pathological conditions ${ }^{2,4}$. Upon TNF signaling, inflammasome activation and cellular stress responses, key pyroptotic and necroptotic pathways, respectively, are initiated and finally result in the abundant release of pro-inflammatory components, including IL-1, IL-18, and damage-associated molecular patterns (DAMPs), which can provoke and augment immune reactions and tissue inflammation ${ }^{1}$. Gasdermin D (Gsdmd) has recently been identified as the executioner of pyroptosis and the cleavage of Gsdmd in its linker domain mediated by inflammasome and specific caspase activation induces its oligomerization and forms pores in the cell plasma membrane, leading to lytic cell death ${ }^{5,6}$. On the other hand, receptor-interacting serine/threonine kinase 3 (Ripk3) is a specific key molecule of necroptosis to activate the pseudokinase mixed lineage kinase domain-like

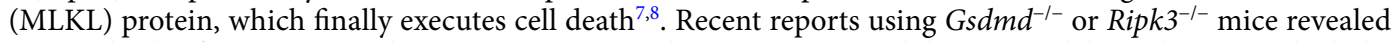
a pivotal role of pyroptosis and necroptosis in several tissue injuries and disease models, such as non-alcoholic hepatitis, familial Mediterranean fever (FMF), experimental autoimmune encephalomyelitis (EAE), ceruleaninduced pancreatitis, ischemia/reperfusion injury, and kidney transplantation ${ }^{9-14}$. Thus, the aberrant activation of programmed cell death exacerbates the tissue inflammation associated with acute injuries and innate cell

${ }^{1}$ Laboratory of Integrative Biological Science, Institute for Frontier Life and Medical Sciences, Kyoto University, 53 Kawahara-cho, Shogoin, Sakyo-ku, Kyoto 606-8507, Japan. ²Department of Experimental Immunology, Immunology Frontier Research Center, Osaka University, Osaka 565-0871, Japan. ${ }^{3}$ Department of Rheumatology and Clinical Immunology, Graduate School of Medicine, Kyoto University, Kyoto 606-8507, Japan. ${ }^{4}$ ljinkai Takeda General Hospital, Kyoto 601-1495, Japan. ${ }^{\boxplus}$ email: hkeiji@infront.kyoto-u.ac.jp 
activation. However, it remains elusive whether programed necrosis plays a crucial role in the pathogenesis of T cell-mediated autoimmune diseases such as rheumatoid arthritis (RA).

RA, one of the most common human autoimmune diseases, affects approximately $1 \%$ of the world population, and is characterized by bone-destructive chronic polyarthritis ${ }^{15}$. Although the precise pathophysiology of RA remains to be determined, it is widely appreciated that $\mathrm{CD} 4^{+} \mathrm{T}$ helper (Th) cells play a pivotal role in the pathogenesis of RA; this is supported by accumulating evidence, for example, MHC class II haplotype human leukocyte antigen (HLA)-DRB1 was identified as the strongest disease susceptibility gene and a high efficacy of cytotoxic T lymphocyte antigen 4 (CTLA4)-Ig blocking T cell co-stimulatory molecules for the treatment of $\mathrm{RA}^{15-17}$. Chronic synovial inflammation can accompany the cell death of proliferated fibroblast-like synoviocytes (FLSs) and recruited immune cells as well as pro-inflammatory cytokine production and the release of DAMPs, presumably by pyroptosis and necroptosis due to various triggers that are present in inflamed joints, including TNF and endogenous inflammasome ligands. Although it is considered that these events can amplify chronic inflammation and joint pathology ${ }^{18}$, it is unclear how a type of programed necrosis contributes to autoimmune arthritis.

SKG mice, a murine model of RA, spontaneously develop IL-17-producing T helper (Th17) cell-dependent autoimmune arthritis, resembling the immunopathology of chronic synovial inflammation in RA patients ${ }^{19,20}$. We have previously shown that DAMPs, including the alarmin IL-33, augment chronic inflammation in the inflamed joints of SKG mice, at least in part by stimulating tissue-resident synovial innate lymphoid cells (ILCs) ${ }^{21}$. Effector Th17 cells together with activated FLSs and ILCs orchestrate chronic arthritis by secreting abundant proinflammatory cytokines, including IL-6, IL-17, GM-CSF, and TNF, and these inflammatory cellular responses and their signaling pathways potentially trigger necrosis. Because lytic forms of programed necrosis can release abundant inflammatory mediators, including DAMPs and IL- $1 \beta$, which is capable of amplifying the autoimmune Th17 effector functions and joint inflammation, in this study, SKG mice were used as a suitable model to investigate whether programed necrosis contributed to the differentiation and expansion of arthritogenic Th17 cells and the development of autoimmune arthritis.

\section{Results}

The generation of $\mathrm{Gsdmd}^{-1-}$ SKG mice and $\mathrm{CD}^{+}{ }^{+} \mathrm{T}$ cell profiles in Peyer's patches under a homeostatic state. To investigate the role of Gsdmd in the pathogenesis of autoimmune arthritis, we first evaluated the Gsdmd expression in whole synovial tissues from healthy and arthritic SKG mice. When arthritis was induced in SKG mice, the expression of Gsdmd tended to be upregulated in the inflamed joints (Fig. 1A). We next assessed the Gsdmd expression in $\mathrm{CD}^{+} \mathrm{T}$ cells, $\mathrm{CD} 11 \mathrm{~b}^{+} \mathrm{Ly}_{6 \mathrm{G}}{ }^{+}$neutrophils, CD11 ${ }^{+} \mathrm{Ly}_{6 \mathrm{G}} \mathrm{Ly}_{6 \mathrm{C}}{ }^{\text {high }}$ inflammatory monocytes and CD $45^{-}$CD $31^{-}$-Podoplanin ${ }^{+}$FLSs sorted from the inflamed joints, each of which are the key cellular components that can augment chronic joint inflammation. The expression of Gsdmd in neutrophils and monocytes was higher in comparison to CD4 ${ }^{+} \mathrm{T}$ cells and FLSs, indicating the possible relevance of Gsdmd in these myeloid cells to the pathophysiology of SKG arthritis (Fig. 1B). We then generated Gsdmd $d^{-1-}$ SKG mice on a BALB/c background by using CRISPR/Cas9 system to examine the effects of Gsdmd on the differentiation and maintenance of $\mathrm{CD}^{+} \mathrm{T}$ cell subsets in homeostatic and inflammatory conditions (Supplementary Fig. 1A). As reported previously in a C57BL/6 background ${ }^{5,10,11}, \mathrm{Gs} \mathrm{bm}^{-1-}$ SKG mice grew up normally like wild-type (WT) SKG mice and the deletion of Gsdmd had no effect on their development, lifespan, or fertility (data not shown). Loss of the Gsdmd function in pyroptosis was confirmed by in vitro assay of LPS transfection into bone marrow-derived dendritic cells (BMDCs) from $G s d m d^{-1-} R_{a g} 2^{-1-}$ mice, which showed a remarkable reduction of the capacity for IL- $1 \beta$ release in comparison to BMDCs from WT Rag $2^{-1-}$ mice (Fig. 1C), as previously reported using other $G s d m d^{-1-}$ strains ${ }^{5}$. To explore whether the physiological Gsdmd expression levels have any impact on $\mathrm{CD} 4^{+} \mathrm{T}$ cell profiles under a homeostatic condition, we assessed the Gsdmd expression in normal tissues from $\mathrm{BALB} / \mathrm{c}$ mice maintained under specific-pathogen-free (SPF) conditions and found that the small intestine is an organ that constitutively expresses the high levels of Gsdmd, and the expression was found to be independent of the gut microbiota and bacterial components based on the comparable expression of Gsdmd in germ-free (GF) mice (Fig. 1D). Because various CD4 ${ }^{+} \mathrm{T}$ cell subsets reside in Peyer's patches of the small intestine, we examined the proportions of physiological Th1, Th17 and regulatory T (Treg) cells in Peyer's patches of WT and Gsdmd $d^{-/-}$ SKG mice under SPF conditions. Gsdmd ${ }^{-1-}$ SKG mice showed almost the same CD4 ${ }^{+} \mathrm{T}$ cell profiles in terms of the expression of IL-17, GM-CSF, IFN- $\gamma$ and Foxp3 in comparison to WT SKG mice (Fig. 1E, F). Taken together, these results indicated that Gsdmd had little impact on the differentiation and maintenance of physiological $\mathrm{CD} 4^{+} \mathrm{T}$ cell subsets under a homeostatic state.

Gsdmd is dispensable for the induction and development of SKG arthritis. We induced arthritis in WT and $G s d m d^{-1-}$ SKG mice and assessed the clinical phenotypes and the development of arthritogenic Th17 cells. The severity of arthritis did not differ between the two groups of mice (Fig. 2A). Notably, the levels of IL-1 $\beta$ in the synovium from the inflamed joints of the two groups were comparable, suggesting that IL-1 $\beta$ could be produced in a Gsdmd-independent mechanism (Fig. 2B). We then assessed the effects of Gsdmd on CD4 $4^{+} \mathrm{T}$ cell profiles after the induction of arthritis. The frequencies of IFN- $\gamma-$, GM-CSF-, IL-17-, and Foxp3-expressing subsets in $\mathrm{CD}^{+} \mathrm{T}$ cells from inflamed joints or draining lymph nodes (LNs) were almost unchanged between the groups (Fig. 2C, D). We also evaluated the onset and severity of arthritis induced by the adoptive transfer of

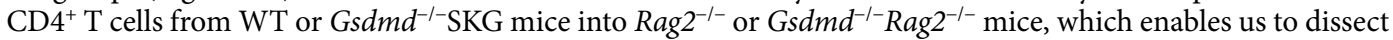
the specific role of Gsdmd in $\mathrm{CD} 4^{+} \mathrm{T}$ cells and non-CD4 ${ }^{+} \mathrm{T}$ cells in recipient mice, separately (Fig. 2E). Like the arthritis phenotype in $G s d m d^{-1-}$ SKG mice, the disease progression curves of arthritis were comparable in the four groups. Furthermore, the differentiation and expansion of arthritogenic Th17 cells in inflamed joints and draining LNs were not affected in Gsdmd-deficient conditions (Fig. 2F-H). These data demonstrated that 
A

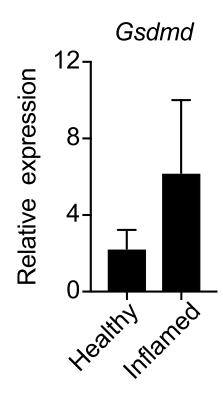

B

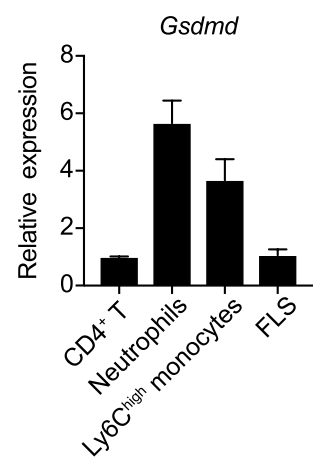

C

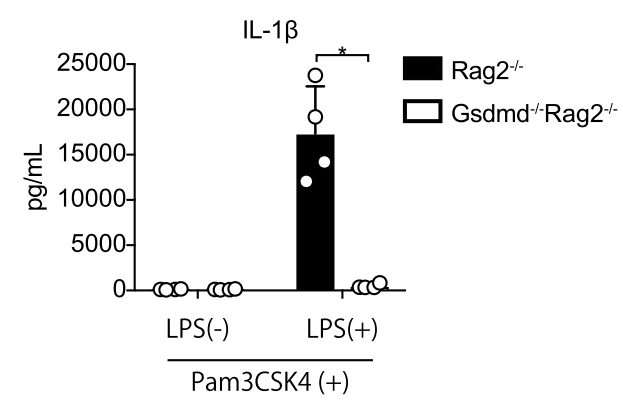

D

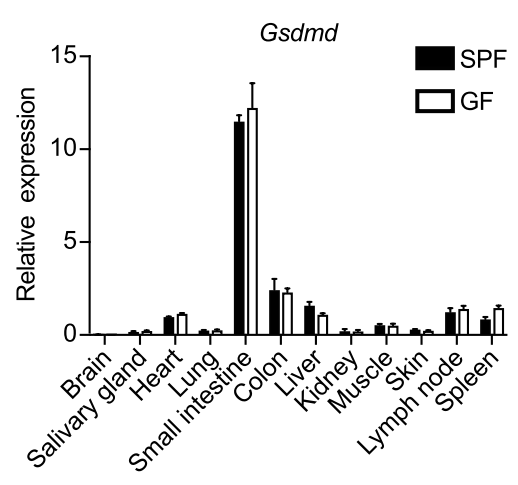

E

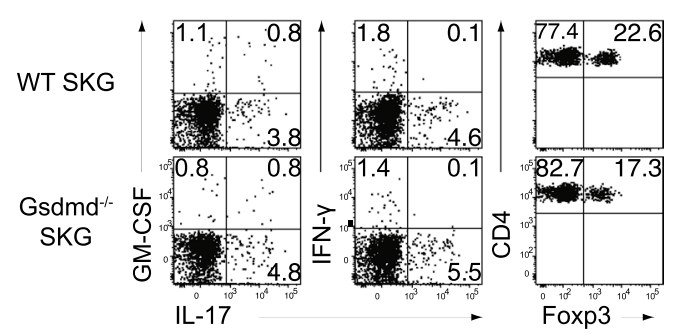

F

Figure 1. The Gsdmd expression in the tissues and inflammatory cells and CD4 + T cell profiles in Gsdmd ${ }^{-1-}$ SKG mice. (A) Quantitative RT-PCR to analyze the expression of Gsdmd in whole synovial tissues of healthy and inflamed joints $(\mathrm{n}=4 \mathrm{each})$. (B) Quantitative RT-PCR to detect the Gsdmd mRNA expression in CD4 ${ }^{+} \mathrm{T}$ cells,

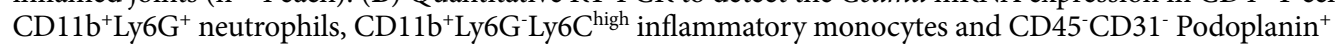
FLS in inflamed joints $(n=4$ each). (C) The IL-1 $\beta$ concentration of culture supernatant of Pam3CSK4-primed BMDCs at $16 \mathrm{~h}$ after LPS transfection $(\mathrm{n}=4 \mathrm{each})$. (D) Quantitative RT-PCR to detect the expression of Gsdmd in the indicated organs of BALB/c mice housed under SPF or germ-free conditions $(n=4$ each). (E,F) Flow cytometry of IL- $17 \mathrm{~A}^{+}, \mathrm{GM}-\mathrm{CSF}^{+}$, IFN- $\gamma^{+}$and Foxp $3^{+}$cells in CD $4^{+} \mathrm{T}$ cells in Peyer's patches of age-matched healthy WT and $G s d m d^{-1-}$ SKG mice $(\mathrm{n}=8 \mathrm{each})$. The vertical bars denote the SD in panel $(\mathbf{A}-\mathbf{C}, \mathbf{F})$ and SEM in panel (D). ${ }^{\star} P<0.05$. Data are pooled from two independent experiments in panels $(\mathbf{A}-\mathbf{D})$ and three independent experiments in panel (F).

Gsdmd is dispensable in the induction of arthritogenic Th17 cells and the development of chronic arthritis in SKG mice.

The generation of Ripk3-l- SKG mice and CD4 ${ }^{+} \mathrm{T}$ cell profiles in Peyer's patches under a homeostatic state. Next, we similarly investigated a role of Ripk3 in CD4 ${ }^{+} \mathrm{T}$ cell homeostasis and the SKG arthritis model. Consistent with the previous report ${ }^{22}$, the expression of Ripk3 was significantly increased in inflamed joints (Fig. 3A). We further assessed the Ripk3 expression in $\mathrm{CD}^{+} \mathrm{T}$ cells, CD $11 \mathrm{~b}^{+} \mathrm{Ly}_{6 \mathrm{G}}{ }^{+}$neutrophils, CD11 ${ }^{+}$Ly6G-Ly6 ${ }^{\text {high }}$ inflammatory monocytes and CD45 CD $31^{-}$Podoplanin ${ }^{+}$FLSs sorted from the inflamed joints. Ripk3 was widely expressed by those key inflammatory cells in SKG arthritic joints, and the expression pattern was different from Gsdmd (Fig. 3B). We then generated Ripk3 ${ }^{-1-}$ SKG mice on a BALB/c background using a CRISPR/Cas9 system to examine the effects of Ripk3 on the differentiation and maintenance of CD4 ${ }^{+}$ $\mathrm{T}$ cell subsets in homeostatic and inflammatory conditions (Supplementary Fig. 1B). Like Gsdmd ${ }^{-/-}$SKG mice, Ripk $^{-1-}$ SKG mice showed no overt phenotypic changes in development or longevity. We confirmed the loss 
A

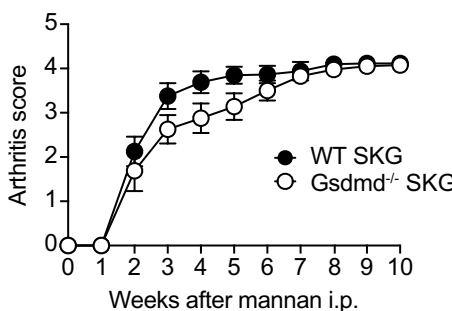

B

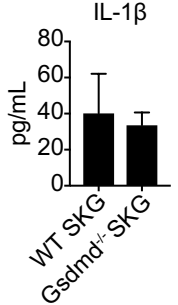

C
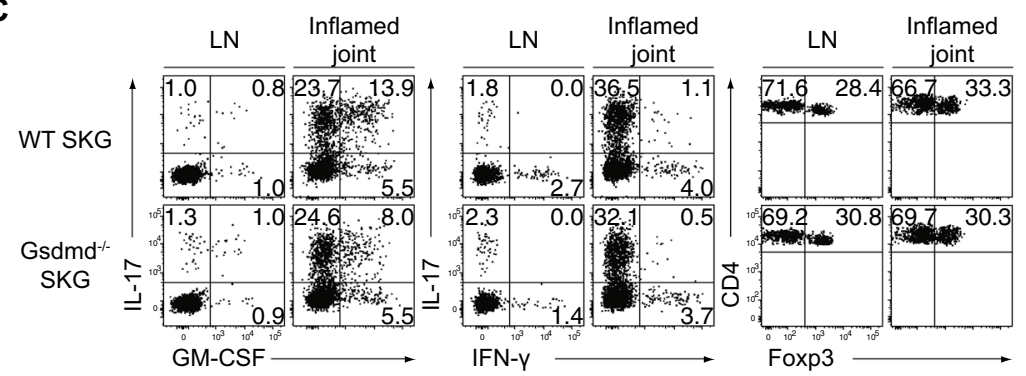

E

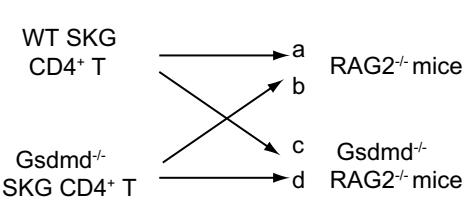

$\mathbf{F}$

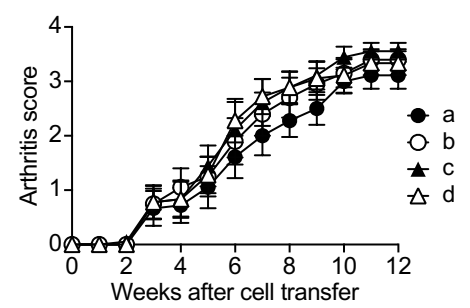

D

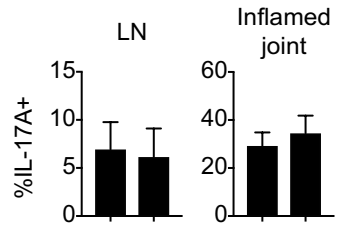

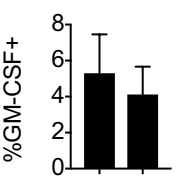

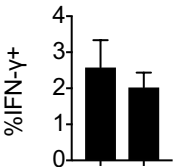

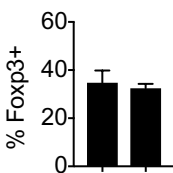

$\cos ^{\mathrm{O}} \mathrm{st}^{\mathrm{O}}$

$a^{2}$ $\left.\begin{array}{r}30 \\ 20 \\ 10 \\ 0\end{array}\right]$ T $T$

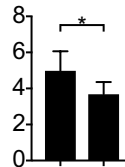

${ }_{20}^{60}{ }_{0}^{6}{ }_{0}{ }^{T}$

St $\mathrm{st}^{\mathrm{T}}$

$2 x_{2}+x^{2}$
G

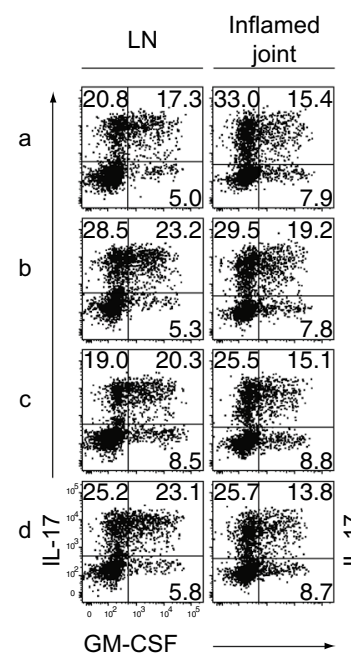

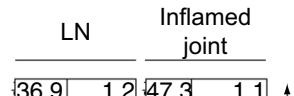

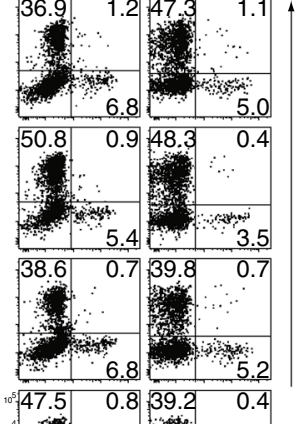

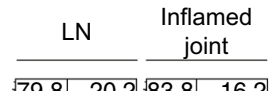

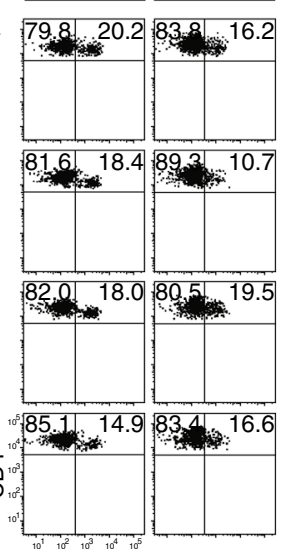

Foxp3

H

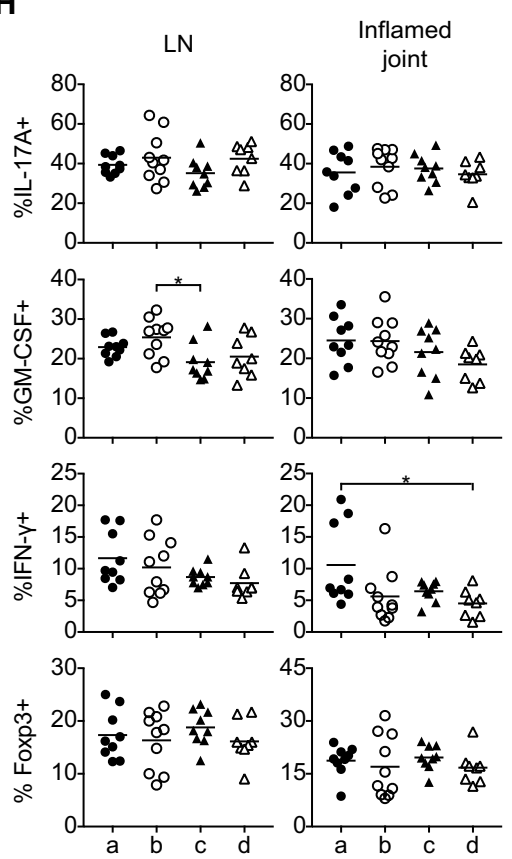

Figure 2. The dispensable role of Gsdmd in the development of autoimmune arthritis. (A) The arthritis scores of WT and $G s d m d^{-1-}$ SKG mice ( $\mathrm{n}=8$ each) after the injection of mannan (20 mg). (B) The IL-1 $\beta$ concentration of synovial tissues from inflamed joints. (C,D) Flow cytometry of IL-17A ${ }^{+}, \mathrm{GM}-\mathrm{CSF}^{+}, \mathrm{IFN}-\gamma^{+}$and Foxp3 $3^{+}$cells in $\mathrm{CD}^{+} \mathrm{T}$ cells in draining LNs and inflamed joints of WT and $G s d m d^{-/-}$SKG mice at 10 weeks after mannan injection ( $\mathrm{n}=8 \mathrm{each}$ ). (E) Experimental design of adoptive transfer of CD4 ${ }^{+} \mathrm{T}$ cells from WT or $G s d m d^{-1-}$

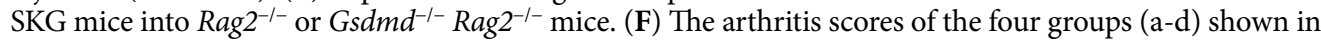

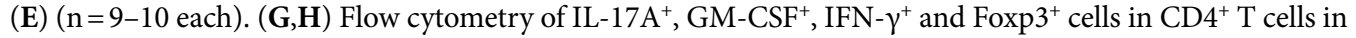
draining LNs and inflamed joints of the four groups of mice at 12 weeks after cell transfer $(n=8-10$ each). The vertical bars denote the SD in panels $(\mathbf{B}, \mathbf{D})$ and SEM in $(\mathbf{A}, \mathbf{F})$. The horizontal line in panel $(\mathbf{H})$ denotes the mean. ${ }^{\star} P<0.05$. Data are pooled from two independent experiments in panels $(\mathbf{A}, \mathbf{B}, \mathbf{D}, \mathbf{F}, \mathbf{H})$. 
A

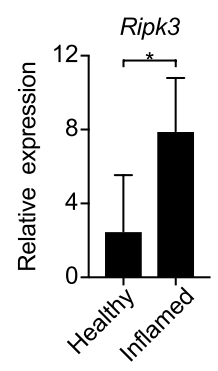

B

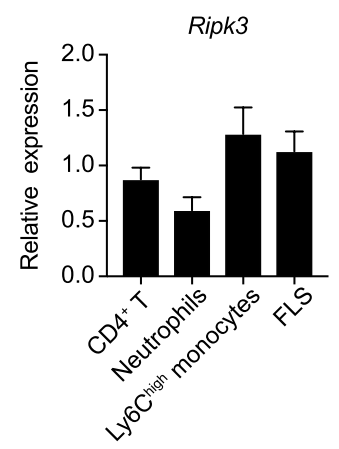

C

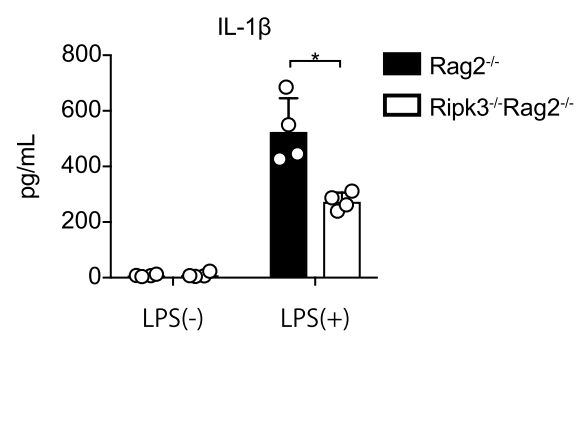

D

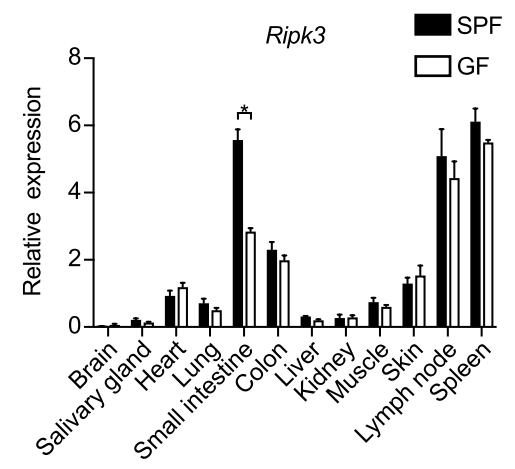

$\mathbf{E}$

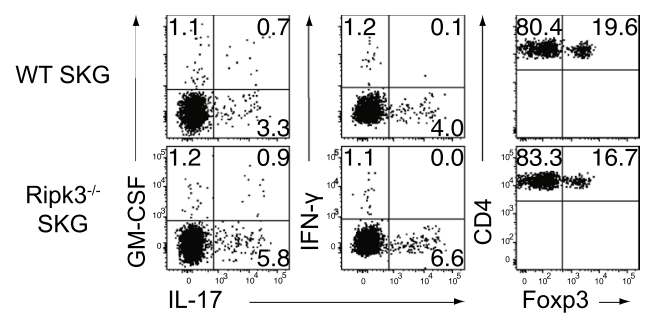

$\mathbf{F}$

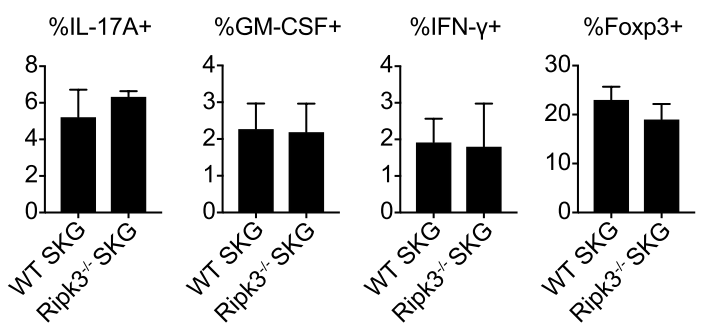

Figure 3. The Ripk3 expression in tissues and inflammatory cells and CD $4+$ T cell profiles in $R i p k 3^{-/-}$SKG mice. (A) Quantitative RT-PCR to detect the expression of Ripk3 in whole synovial tissues of healthy and inflamed joints $\left(\mathrm{n}=4\right.$ each). (B) Quantitative RT-PCR to detect the Ripk 3 mRNA expression in CD ${ }^{+} \mathrm{T}$ cells, CD $11 b^{+}{\text {Ly } 6 G^{+}}$neutrophils, CD11 b ${ }^{+}$Ly6G Ly6C ${ }^{\text {high }}$ inflammatory monocytes and CD $45^{-}$CD $31^{-}$Podoplanin ${ }^{+}$ FLS in inflamed joints $(\mathrm{n}=4 \mathrm{each})$. (C) The IL- $1 \beta$ concentration of culture supernatant of BMDCs at $16 \mathrm{~h}$ after LPS stimulation ( $\mathrm{n}=4$ each) (D) Quantitative RT-PCR to detect the expression of Ripk3 in the indicated organs of BALB/c mice housed under SPF or GF conditions $\left(n=4\right.$ each). (E,F) Flow cytometry of IL-17A , GM-CSF $^{+}$, IFN- $\gamma^{+}(\mathrm{n}=3$ each $)$ and Foxp $3^{+}$cells $(\mathrm{n}=4$ each $)$ in CD4 ${ }^{+} \mathrm{T}$ cells in Peyer's patches of WT and Ripk $3^{-1-}$ SKG mice. The vertical bars denote the SD in panel $(\mathbf{A}-\mathbf{C}, \mathbf{F})$ and SEM in panel (D). ${ }^{\star} P<0.05$. Data are pooled from two independent experiments in panels $(\mathbf{A}-\mathbf{D}, \mathbf{F})$.

of the Ripk3 function in necroptosis by culturing BMDCs from Ripk3 ${ }^{-1-} \mathrm{Rag}^{-1-}$ mice with LPS, which showed

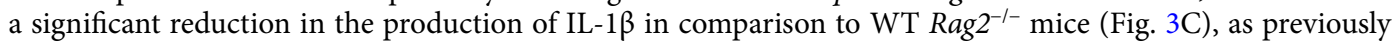
reported $^{23}$. When the physiological Ripk3 expression levels in normal tissues from BALB/c mice maintained under SPF or GF conditions were assessed, the Ripk3 expression was relatively high in the gut, LNs, and spleen (Fig. 3D). Among them, the expression of Ripk3 in the small intestine was likely controlled by the gut microbiota because of the specific reduction of its expression in GF mice (Fig. 3D). However, we found no difference in the frequency of the IL-17A, GM-CSF, IFN- $\gamma$ and Foxp3 expression in the CD4 ${ }^{+} \mathrm{T}$ cells of Peyer's patches between WT and Ripk $3^{-1-}$ SKG mice (Fig. 3E, F). Taken together, these data indicated that Ripk3 did not affect the differentiation or maintenance of physiological $\mathrm{CD} 4^{+} \mathrm{T}$ cell subsets under a homeostatic state.

Ripk3 is dispensable for the induction and development of SKG arthritis. We next investigated the role of Ripk3 in the induction and development of SKG arthritis. We induced arthritis in WT and Ripk $3^{-1-}$ SKG mice. There was no significant difference in the clinical arthritis scores of the two groups of mice (Fig. 4A). Unlike the in vitro assay, IL- $1 \beta$ could be secreted in the inflamed synovium of Ripk $3^{-1-}$ SKG mice (Fig. 4B). The frequencies of IFN- $\gamma-$, GM-CSF-, IL-17-, and Foxp3-expressing subsets in CD4 ${ }^{+} \mathrm{T}$ cells in inflamed joints and draining LNs were almost comparable between the groups (Fig. 4C, D). In addition, adoptive transfer experi- 
A

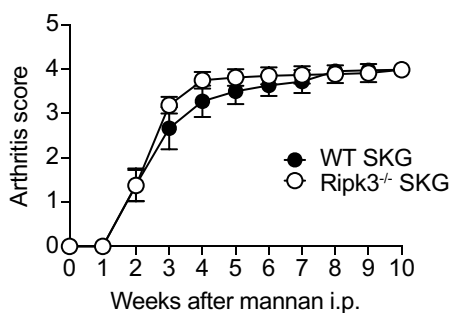

B

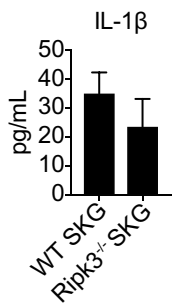

C

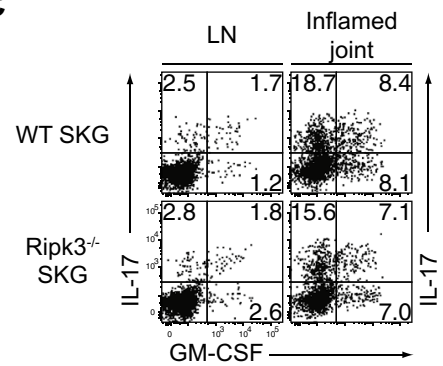

E

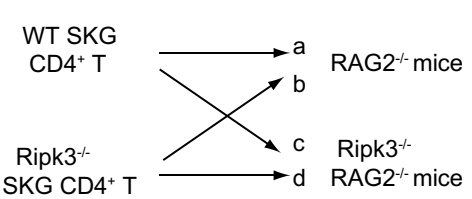

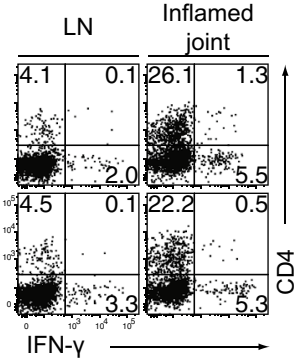

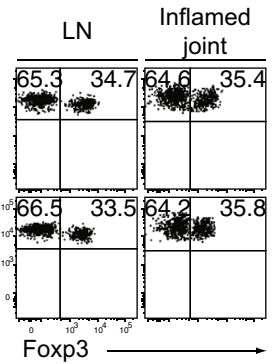

$\mathbf{F}$

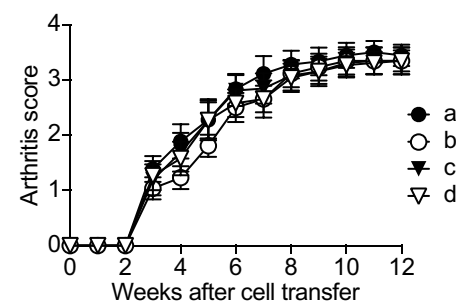

D

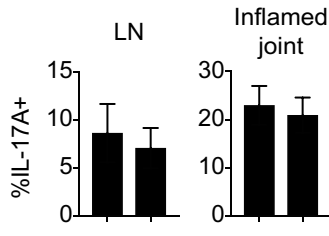

$\left.\begin{array}{cr} & 15 \\ \text { 岁 } & 10 \\ \sum_{0}^{1} & 5 \\ 0 & 0\end{array}\right] T_{1}$

$\left.\begin{array}{l}30 \\ 20 \\ 10 \\ 0\end{array}\right] \stackrel{*}{ }$

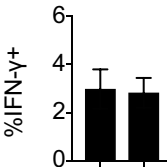

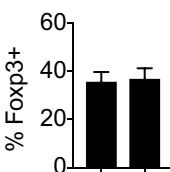
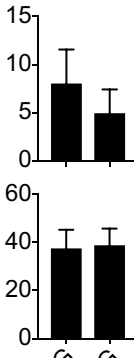

sto $^{\mathrm{C}^{0}} \mathrm{st}^{\mathrm{O}}$

isis<smiles>[C-]1C=[C-]O1</smiles>

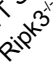

G

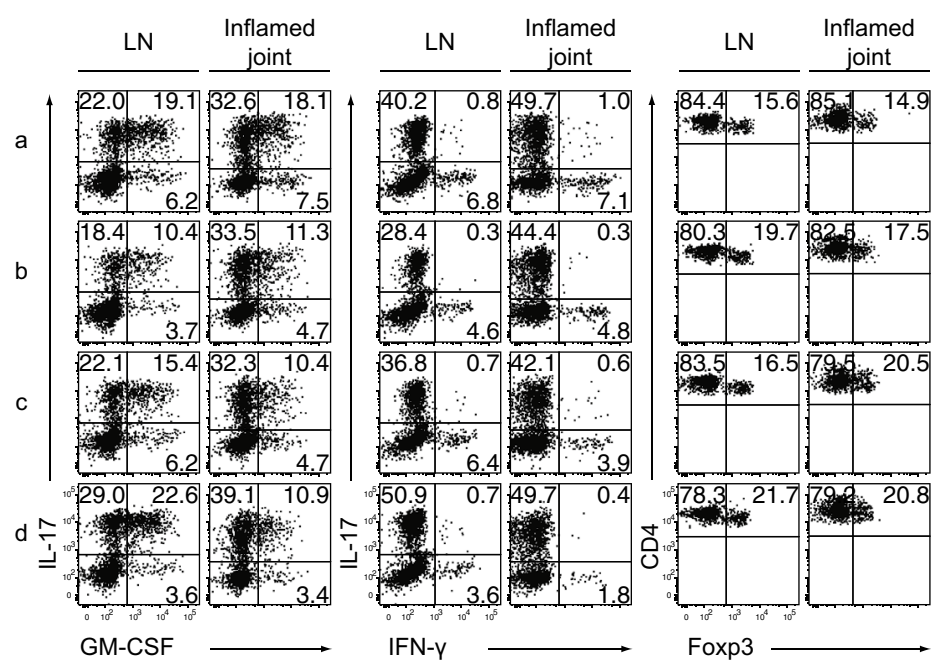

H

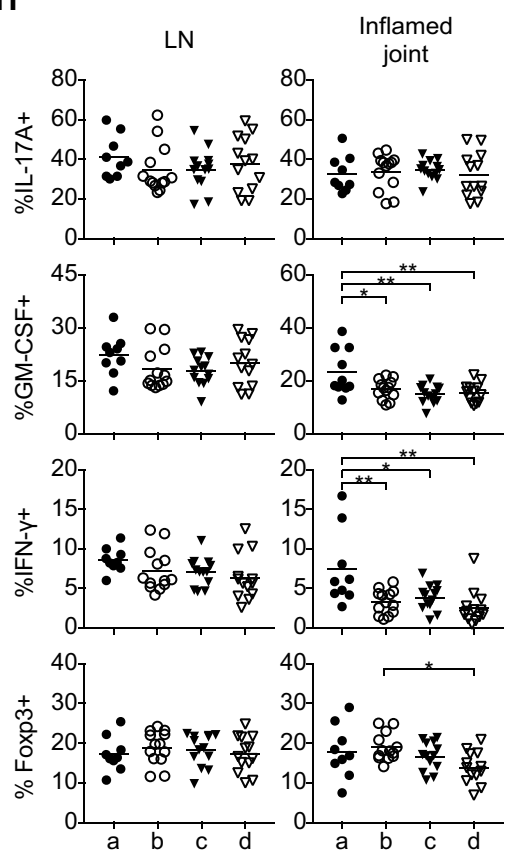

Figure 4. The dispensable role of Ripk3 in the development of autoimmune arthritis. (A) Arthritis scores of WT ( $\mathrm{n}=9)$ and Ripk ${ }^{-1-}$ SKG mice $(\mathrm{n}=8)$ after the injection of mannan $(20 \mathrm{mg})$. (B) The IL- $1 \beta$ concentration of synovial tissues from inflamed joints. (C,D) Flow cytometry of IL- $17 \mathrm{~A}^{+}, \mathrm{GM}-\mathrm{CSF}^{+}$, IFN- $\gamma^{+}$and Foxp3 ${ }^{+}$ cells in $\mathrm{CD}^{+} \mathrm{T}$ cells in draining LNs and the inflamed joints of WT $(\mathrm{n}=9)$ and Ripk $3^{-/-}$SKG mice $(\mathrm{n}=8)$ at 10 weeks after mannan injection. (E) Experimental design of the adoptive transfer of CD4 ${ }^{+} \mathrm{T}$ cells from WT or

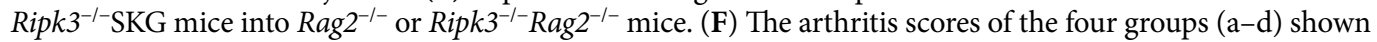
in (E) $(n=9$ or 13$)$. $(\mathbf{G}, \mathbf{H})$ Flow cytometry of IL- $17 \mathrm{~A}^{+}, \mathrm{GM}-\mathrm{CSF}^{+}, \mathrm{IFN}-\gamma^{+}$and Foxp3 $3^{+}$cells in CD $4^{+} \mathrm{T}$ cells in draining LNs and inflamed joints of the four groups of mice 12 weeks after cell transfer $(n=9$ or 13). The vertical bars denote the SD in panels $(\mathbf{B}, \mathbf{D})$ and SEM in $(\mathbf{A}, \mathbf{F})$. The horizontal line in panel $(\mathbf{H})$ denotes the mean. ${ }^{\star} P<0.05,{ }^{* *} P<0.01$. Data are pooled from two independent experiments in panels $(\mathbf{A}, \mathbf{B}, \mathbf{D}, \mathbf{F}, \mathbf{H})$. 
ments using $\mathrm{CD}^{+} \mathrm{T}$ cells from WT or Ripk $3^{-/-}$SKG mice also revealed no significant differences in the clinical arthritis scores or the proportions of arthritogenic Th17 cells in draining LNs and inflamed joints from the

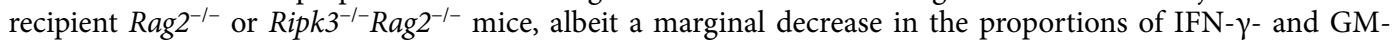
CSF-producing Th cells in the inflamed joints (Fig. 4E-H). Taken together, these data indicated that Ripk3 is dispensable in the induction of pathogenic Th17 cells and the development of chronic arthritis in SKG mice.

Crosstalk or the compensatory role between Gsdmd and Ripk3 is not required for the induction and development of SKG arthritis. Because a compensatory role and context-dependent interaction between Gsdmd and Ripk3 have been described ${ }^{24,25}$, we generated Gsdmd ${ }^{-1-}$ Ripk $^{-1-}$ SKG mice by crossing Gsdmd ${ }^{-1-}$ SKG and Ripk3 ${ }^{-1-}$ SKG mice to assess whether Ripk3 or Gsdmd could compensate for a Gsdmd- or Ripk3-deficient condition, respectively, in the development of autoimmune arthritis, and evaluated their arthritis phenotypes and the development of Th17 cells in Gsdmd and Ripk3 double-deficient conditions. The arthritis scores of Gsdmd $d^{-1-}$ Ripk3 $^{-1-}$ SKG mice after the induction of arthritis were unchanged from those in WT SKG mice (Fig. 5A). The differentiation and expansion of IFN- $\gamma-$, GM-CSF-, and IL-17-producing Th subsets from

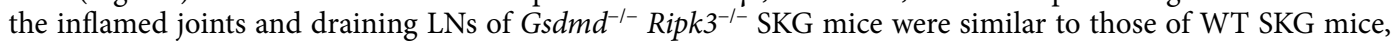
albeit with a marginal decrease in the proportions of Foxp $3^{+}$Treg cells (Fig. 5B, C). Collectively, these results further strengthened the concept that both Gsdmd and Ripk3 and their interactions are dispensable for the induction and development of chronic autoimmune arthritis in SKG mice.

\section{Discussion}

Although accumulated evidence strongly supports that pyroptosis and necroptosis have evolved to combat against infectious pathogens that escape detection by infection-induced apoptosis, particularly intracellular bacteria such as Shigella flexneri, the subsequent acute tissue inflammation and pro-inflammatory cytokine production triggered by the programmed cell death could mediate the immunopathology through the aberrant activation of various inflammatory cells ${ }^{1-4,9-14}$. It is noteworthy that the Ripk3 and MLKL-molecules that are specifically involved in necroptosis-were reported to be upregulated in the inflamed synovium of collageninduced arthritis, indicating a possible contribution of necroptosis to the formation of severe synovitis ${ }^{22}$. How programmed necrosis contributes to T cell-mediated autoimmune arthritis remains poorly understood. Thus, one of the key questions we addressed in the present study was whether the pyroptosis and necroptosis pathways would exacerbate chronic inflammation, in particular sterile synovial inflammation. Using Gsdmd ${ }^{-I-}, R_{i p k 3^{-I-}}$, or $G s d m d^{-1-}$ Ripk $^{-1-}$ mice, we demonstrated that despite abundant cell death in inflamed joints, pyroptosis and necroptosis are not required for the expansion of arthritogenic Th17 cells or the progression of Th17-mediated autoimmune arthritis.

Inflammatory mediators, including DAMPs and pro-inflammatory cytokines released by pyroptosis and necroptosis could amplify Th17 functions; however, their modulation of pathogenic Th17 cells seems to be dependent on the type of inflammation and the target organ. Lytic forms of cell death seemed to augment systemic auto-inflammation in an FMF model, and acute tissue injuries in ischemia and transplantation models through the excess production of IL- $1 \beta$ and DAMPs, which could mediate pathology by the activation of innate immune cells ${ }^{10,12-14}$. Given that the pathogenesis of these models is less dependent on adaptive T cell functions, pyroptosis and necroptosis are probably involved in the exacerbation of acute inflammation and tissue injuries are often autonomously amplified by the activation of innate immune and mesenchymal cells. On the other hand, the role of pyroptosis and necroptosis in T cell-mediated autoimmune pathology may be dispensable or limited in a specific inflammatory context. EAE is an exceptional model which relies on Gsdmd for the development of Th17-mediated autoimmune neuroinflammation. Mechanistically, Gsdmd-dependent pyroptosis of the peripheral myeloid cells is necessary for the activation and differentiation of pathogenic Th cells in peripheral lymphoid tissues, which in turn migrate into the target spinal cord to cause chronic tissue inflammation ${ }^{11}$. Although pathogenic Th17 cells predominantly drive SKG arthritis and EAE, the differential requirement of Gsdmd in the models may be attributed how these diseases are induced. For EAE, immunization with complete Freund's adjuvant and pertussis toxin, potent bacterial compounds, may be a strong inducer of pyroptosis in myeloid cells, following the activation of inflammasomes. In contrast, SKG mice are a spontaneous arthritis model and can be induced by the injection of mannan, which is a fungal compound distinct from that used in EAE and may not be involved in the activation of the pyroptosis pathway. Therefore, $\mathrm{T}$ cell priming in peripheral LNs between the models is probably different and Gsdmd was not required for the induction of arthritogenic Th17 cells in the LNs. However, it is tempting to speculate that persistent IL- $1 \beta$ production and Th17-mediated chronic inflammation in the target tissues of EAE or SKG mice may be similarly independent of pyroptosis and necroptosis, despite abundant cell death occurring in the inflamed tissue.

Crosstalk among different forms of programmed cell death, including apoptosis and necrosis, has recently been highlighted as a new aspect of interactions and compensatory roles between the signaling pathways of the distinct programs ${ }^{24-26}$. Although there are various triggers (TNF, endogenous inflammasome ligands, etc.)

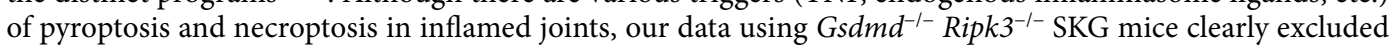
the compensatory role between Gsdmd and Ripk3 for the development of autoimmune arthritis and further strengthened our claim that they are not involved in Th17 cell-mediated chronic inflammation. However, con-

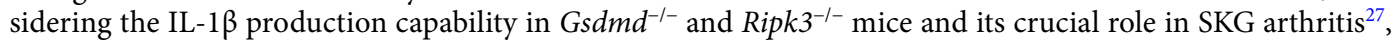
unappreciated cellular mechanisms that can also process IL-1 $\beta$ maturation and release may be present in sterile tissue inflammation (e.g., the synovium and central nervous system) ${ }^{28,29}$.

In contrast, given that $G s d m d^{-1-}$ and Ripk $3^{-1-}$ mice do not show an overt phenotype in development or physiology, inhibitors of Gsdmd or Ripk3 may be a potential therapeutic target for the treatment of innate immune cell-mediated tissue injuries and systemic auto-inflammation because the inhibitors could have limited adverse 
A

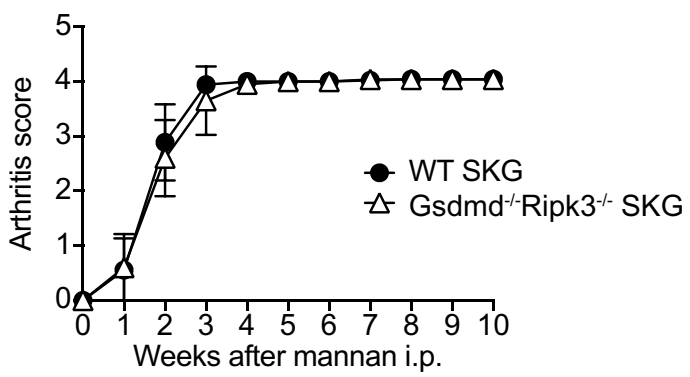

B

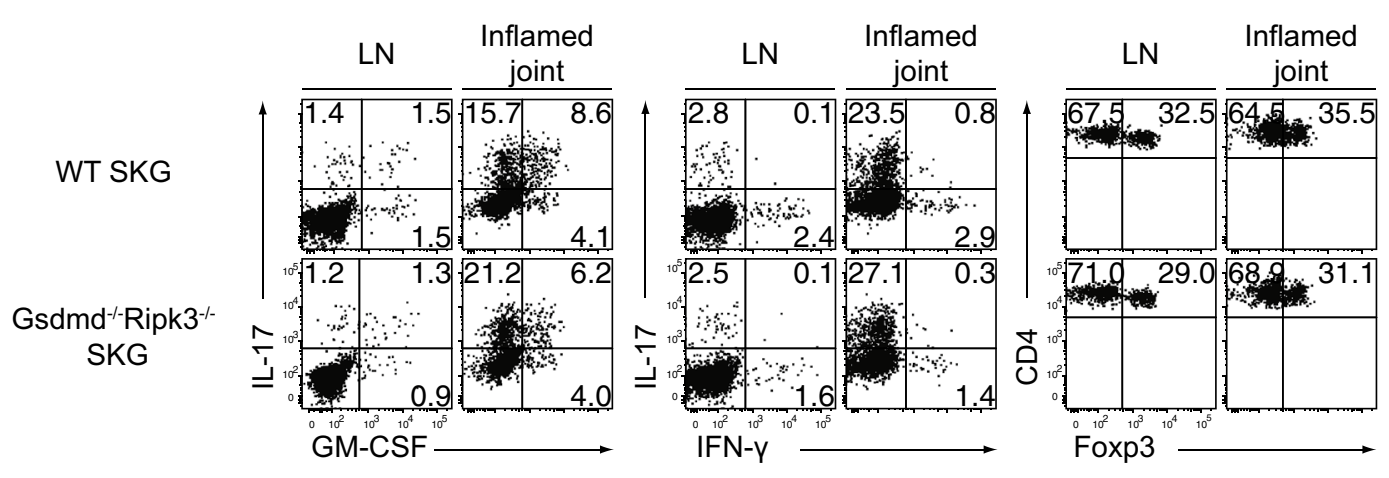

C

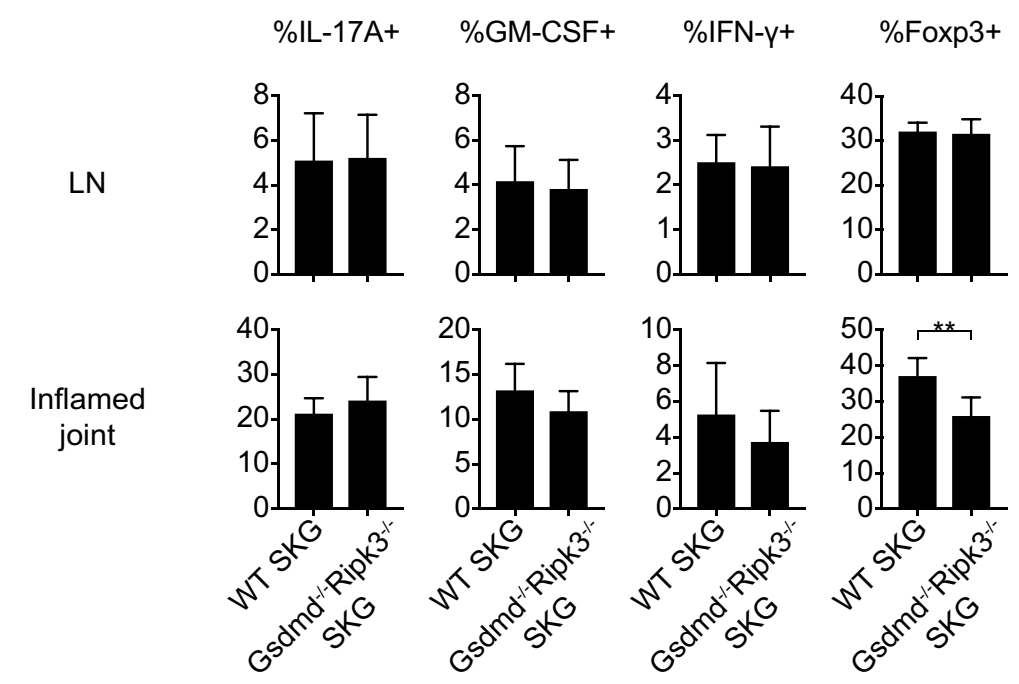

Figure 5. The dispensable role of crosstalk between Gsdmd and Ripk 3 in the development of autoimmune

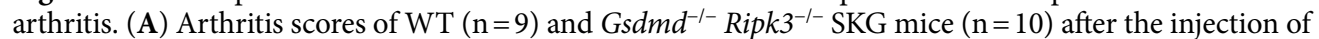
mannan $(20 \mathrm{mg})$. (B,C) Flow cytometry of IL- $17 \mathrm{~A}^{+}, \mathrm{GM}-\mathrm{CSF}^{+}$, IFN- $\gamma^{+}$and Foxp $3^{+}$cells in $\mathrm{CD}^{+} \mathrm{T}$ cells in draining LNs and inflamed joints of WT $(\mathrm{n}=9)$ and $\mathrm{Gsdmd}^{-1-} \mathrm{Ripk}^{-/-}$SKG mice $(\mathrm{n}=10)$. The vertical bars denote the SD in panel $(\mathbf{C})$ and the SEM in $(\mathbf{A}) .{ }^{*} P<0.01$. Data are pooled from two independent experiments in panels $(\mathbf{A}, \mathbf{C})$.

effects with regard to the blocking of physiological functions and $\mathrm{T}$ cell-mediated immunity. Further studies are needed to investigate the unknown mechanisms through which programmed cell death modalities are involved in the production of pro-inflammatory mediators (e.g., IL-1ß), and the amplification of chronic tissue inflammation in autoimmune diseases. 


\section{Materials and methods}

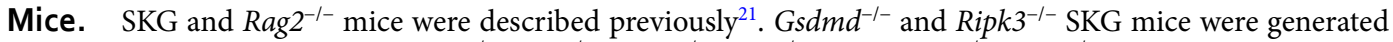

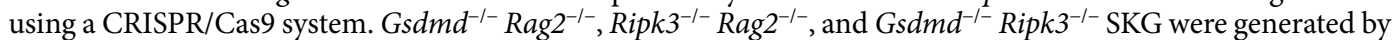
crossing the above described strains. BALB/c mice housed under SPF and GF conditions were purchased from CLEA Japan. All animal experiments were approved by the Ethical Committee of Institute for Frontier Life and Medical Sciences and Graduate School of Medicine, Kyoto University, and were performed in compliance with the institutional and ARRIVE guidelines.

Induction of arthritis. Arthritis was induced by a single intraperitoneal injection of mannan (20 mg; Sigma-Aldrich) in SKG mice, or by adoptive transfer of SKG CD4 ${ }^{+} \mathrm{T}$ cells (intravenously) into recipient mice, and was scored as described previously ${ }^{19,20,30}$. For the adoptive transfer of CD4 ${ }^{+} \mathrm{T}$ cells, CD $4^{+} \mathrm{T}$ cells were positively selected from the spleen and peripheral LNs using MACS CD4 microbeads and an LS column (Miltenyi Biotec) according to the manufacturer's instruction, and $2 \times 10^{6} \mathrm{CD} 4^{+} \mathrm{T}$ cells were transferred into each recipient mouse intravenously via the tail vein.

Single cell suspension from synovial tissue and flow cytometry. The collected synovial tissues were cut into small pieces, followed by enzymatic digestion for $1 \mathrm{~h}$ at $37^{\circ} \mathrm{C}$ in $3 \mathrm{ml}$ of IMDM buffer containing $2 \%$ FBS (GIBCO) with $300 \mathrm{U}$ of collagenase I and collagenase IV (Worthington). Digested tissues were then mashed through a $70-\mu \mathrm{m}$ mesh filter. The resultant single cell suspension of the synovial tissues was used for flow cytometry and cell sorting experiments. For intracellular staining of cytokines, cells were restimulated for $2.5 \mathrm{~h}$ with phorbol 12-myristate 13 -acetate $(50 \mathrm{ng} / \mathrm{ml}$; Sigma-Aldrich) and ionomycin ( $500 \mathrm{ng} / \mathrm{ml}$; Sigma-Aldrich) in the presence of Brefeldin A (1 $\mu \mathrm{g} / \mathrm{ml}$; Merck) in IMDM (Sigma-Aldrich) supplemented with 5\% FBS (GIBCO), 2-mercaptoethanol (GIBCO), GlutaMAX (GIBCO), sodium pyruvate (GIBCO), MEM NEAA (GIBCO) and penicillin-streptomycin (Nacalai Tesque). Cells were then fixed with $3.7 \%$ formaldehyde (Sigma-Aldrich), followed by permeabilization with $0.1 \%$ NP-40 (Nacalai Tesque), and staining with antibodies against cytokines. Foxp3 was stained using the Foxp3/Transcription Factor Staining Buffer Set (eBioscience) in accordance with the manufacturer's instructions. The following monoclonal antibodies were used for flow cytometry (BD FACSCantoII) and cell sorting (SONY MA900): anti-mouse CD4 (RM4-4), CD11b (M1/70), CD45.2 (104), Ly-6G (1A8), Podoplanin (8.1.1), IL-17A (TC11-18H10.1), GM-CSF (MP1-22E9), and IFN- $\gamma$ (XMG1.2), all from Biolegend, anti-mouse Ly-6C (AL-21), CD31 (390) and PE-Streptavidin, all from BD Bioscience, anti-mouse Foxp3 (FJK-16 s) from eBioscience. All FACS data were analyzed using the FlowJo software program (Tree Star, Inc.).

Quantitative RT-PCR. Total RNA from tissues and sorted cells was extracted using TRIzol (InvitroGen) and was reverse-transcribed with SuperScript VILO (Invitrogen). The resultant cDNA was used for quantitative RT-PCR on a Light cycler 480 (Roche) or StepOnePlus (Applied Biosystems) using qPCR master mix (TOYOBO) or Luna Universal qPCR Master Mix (NEB) and TaqMan Gene Expression Assays (Thermo Fisher: Gsdmd, Mm00509958_m1; Ripk3 Mm00444947_m1; Hprt, Mm03024075_m1). The expression levels of target genes were quantified after normalization to the expression of Hprt as a housekeeping gene.

Generation of bone marrow-derived dendritic cells (BMDCs). Bone marrow cells were collected from the femur and tibia and red blood cells were lysed with RBC lysis buffer (Sigma-Aldrich). Resultant cells were cultured at $5 \times 10^{5} / \mathrm{ml}$ in RPMI containing $10 \% \mathrm{FBS}$, 2-mercaptoethanol and penicillin-streptomycin with $20 \mathrm{ng} / \mathrm{ml} \mathrm{GM-CSF}$ for 6 days to differentiate into BMDCs for in vitro assays.

Measurement of IL-1 $\beta$ from BMDCs and joint tissues. BMDCs were cultured in 96-well plates at $1 \times 10^{5}$ cells per well and either treated with $1 \mu \mathrm{g} / \mathrm{ml}$ LPS (InvivoGen), or transfected with $1 \mu \mathrm{g} / \mathrm{ml} \mathrm{LPS}$ using $0.3 \% \mathrm{v} / \mathrm{v}$ FuGENE HD (Promega) after priming with $1 \mu \mathrm{g} / \mathrm{ml}$ Pam3CSK4 (InvivoGen) for $6 \mathrm{~h}$. At $16 \mathrm{~h}$ after LPS stimulation, the IL- $1 \beta$ concentrations in the culture supernatants were measured using a Cytometric Bead Array (BD Biosciences) in accordance with the manufacturer's instructions. As for the measurement of IL-1 $\beta$ in the synovium, synovial tissues were resected from the inflamed joints, cut into small pieces, and the tissue weight was measured. The resultant synovial tissues were placed into $500 \mu \mathrm{l}$ of D-PBS (-) (Nacalai Tesque), then vortexed and centrifuged. The collected supernatants were then used for the measurement of the IL- $1 \beta$ concentration, which was performed using a Cytometric Bead Array, and the results were normalized with tissue weights of individual samples.

Statistical analysis. Statistical analyses were performed using the GraphPad PRISM7 software program (GraphPad Software). A two-tailed t-test was used for the statistical analysis. A two-way ANOVA followed by Tukey's multiple comparisons test was used for the analysis of grouped data. Data are shown as the mean \pm standard error of mean (SEM) or mean \pm standard deviation (SD). $P$ values of $<0.05$ were considered to indicate statistical significance.

\section{Data availability}

The datasets analyzed during the current study are available from the corresponding author on reasonable request.

Received: 27 April 2021; Accepted: 26 August 2021

Published online: 21 September 2021 


\section{References}

1. Frank, D. \& Vince, J. E. Pyroptosis versus necroptosis: Similarities, differences, and crosstalk. Cell Death Differ. 26, 99-114 (2019).

2. Orning, P., Lien, E. \& Fitzgerald, K. A. Gasdermins and their role in immunity and inflammation. J. Exp. Med. 216, 2453-2465 (2019).

3. Orzalli, M. H. \& Kagan, J. C. Apoptosis and necroptosis as host defense strategies to prevent viral infection. Trends Cell Biol. 27, 800-809 (2017).

4. Khoury, M. K., Gupta, K., Franco, S. R. \& Liu, B. Necroptosis in the pathophysiology of disease. Am. J. Pathol. 190, 272-285 (2020).

5. Kayagaki, N. et al. Caspase-11 cleaves gasdermin D for non-canonical inflammasome signalling. Nature 526, 666-671 (2015).

6. Shi, J. et al. Cleavage of GSDMD by inflammatory caspases determines pyroptotic cell death. Nature 526, 660-665 (2015).

7. Sun, L. et al. Mixed lineage kinase domain-like protein mediates necrosis signaling downstream of RIP3 kinase. Cell 148, 213-227 (2012).

8. Murphy, J. M. et al. The pseudokinase MLKL mediates necroptosis via a molecular switch mechanism. Immunity 39, 443-453 (2013).

9. $\mathrm{Xu}, \mathrm{B}$. et al. Gasdermin D plays a key role as a pyroptosis executor of non-alcoholic steatohepatitis in humans and mice. J. Hepatol. 68, 773-782 (2018).

10. Kanneganti, A. et al. GSD MD is critical for autoinflammatory pathology in a mouse model of familial mediterranean fever. J. Exp. Med. 215, 1519-1529 (2018).

11. Li, S. et al. Gasdermin D in peripheral myeloid cells drives neuroinflammation in experimental autoimmune encephalomyelitis. J. Exp. Med. 216, 2562-2581 (2019).

12. He, S. et al. Receptor interacting protein kinase-3 determines cellular necrotic response to TNF-a. Cell 137, 1100-1111 (2009).

13. Zhang, T. et al. CaMKII is a RIP3 substrate mediating ischemia- and oxidative stress-induced myocardial necroptosis. Nat. Med. 22, 175-182 (2016).

14. Lau, A. et al. RIPK3-mediated necroptosis promotes donor kidney inflammatory injury and reduces allograft survival. Am. J. Transplant. 13, 2805-2818 (2013).

15. Smolen, J. S., Aletaha, D. \& McInnes, I. B. Rheumatoid arthritis. Lancet 388, 2023-2038 (2016).

16. Gregersen, P. K., Silver, J. \& Winchester, R. J. The shared epitope hypothesis. Arthritis Rheum. 30, 1205-1212 (1987).

17. Gonzalez-Gay, M. A., Garcia-Porrua, C. \& Hajeer, A. H. Influence of human leukocyte antigen-DRB1 on the susceptibility and severity of rheumatoid arthritis. Semin. Arthritis Rheum. 31, 355-360 (2002).

18. Anderton, H., Wicks, I. P. \& Silke, J. Cell death in chronic inflammation: Breaking the cycle to treat rheumatic disease. Nat. Rev. Rheumatol. 16, 496-513 (2020).

19. Sakaguchi, N. et al. Altered thymic T-cell selection due to a mutation of the ZAP-70 gene causes autoimmune arthritis in mice. Nature 426, 454-460 (2003).

20. Hirota, K. et al. T cell self-reactivity forms a cytokine milieu for spontaneous development of IL-17+ Th cells that cause autoimmune arthritis. J. Exp. Med. 204, 41-47 (2007).

21. Hirota, K. et al. Autoimmune Th17 cells induced synovial stromal and innate lymphoid cell secretion of the cytokine GM-CSF to initiate and augment autoimmune arthritis. Immunity 48, 1220-1232.e5 (2018).

22. Lee, S. H., Kwon, J. Y., Kim, S. Y., Jung, K. A. \& Cho, M. L. Interferon-gamma regulates inflammatory cell death by targeting necroptosis in experimental autoimmune arthritis. Sci. Rep. 7, 2-10 (2017).

23. Moriwaki, K., Bertin, J., Gough, P. J. \& Chan, F.K.-M. A RIPK3-caspase 8 complex mediates atypical Pro-IL-1 $\beta$ Processing. J. Immunol. 194, 1938-1944 (2015).

24. Gutierrez, K. D. et al. MLKL activation triggers NLRP3-mediated processing and release of IL-1 $\beta$ independently of gasdermin-D. J. Immunol. 198, 2156-2164 (2017).

25. Conos, S. A. et al. Active MLKL triggers the NLRP3 inflammasome in a cell-intrinsic manner. Proc. Natl. Acad. Sci. USA 114, E961-E969 (2017).

26. Doerflinger, M. et al. Flexible usage and interconnectivity of diverse cell death pathways protect against intracellular infection. Immunity 53, 533-547.e7 (2020).

27. Hata, H. et al. Distinct contribution of IL-6, TNF- $\alpha$, IL-1, and IL-10 to T cell-mediated spontaneous autoimmune arthritis in mice. J. Clin. Invest. 114, 582-588 (2004).

28. Conos, S. A., Lawlor, K. E., Vaux, D. L., Vince, J. E. \& Lindqvist, L. M. Cell death is not essential for caspase-1-mediated interleukin-1 $\beta$ activation and secretion. Cell Death Differ. 23, 1827-1838 (2016).

29. Rashidi, M. et al. The pyroptotic cell death effector gasdermin D is activated by gout-associated uric acid crystals but is dispensable for cell death and IL-1 $\beta$ release. J. Immunol. 203, 736-748 (2019).

30. Hashimoto, M. et al. Complement drives Th17 cell differentiation and triggers autoimmune arthritis. J. Exp. Med. 207, 1135-1143 (2010).

\section{Acknowledgements}

This work was supported by a JSPS Grant-in-Aid for Scientific Research (19H01026 to KH), the Takeda Science Foundation (KH), the Mochida Memorial Foundation for Medical and Pharmaceutical Research (KH), and the Uehara Memorial Foundation (KH).

\section{Author contributions}

Y.T. and K.H. designed the study. Y.T., D.O., H.W., G.K., and K.H. performed the experiments. N.S., S.S., A.M., and T.M. provided intellectual input. Y.T. and K.H. wrote the manuscript. K.H. supervised the project. All authors reviewed the manuscript.

\section{Competing interests}

The authors declare no competing interests.

\section{Additional information}

Supplementary Information The online version contains supplementary material available at https://doi.org/ 10.1038/s41598-021-98145-y.

Correspondence and requests for materials should be addressed to K.H.

Reprints and permissions information is available at www.nature.com/reprints.

Publisher's note Springer Nature remains neutral with regard to jurisdictional claims in published maps and institutional affiliations. 
(c) (i) Open Access This article is licensed under a Creative Commons Attribution 4.0 International cc) License, which permits use, sharing, adaptation, distribution and reproduction in any medium or format, as long as you give appropriate credit to the original author(s) and the source, provide a link to the Creative Commons licence, and indicate if changes were made. The images or other third party material in this article are included in the article's Creative Commons licence, unless indicated otherwise in a credit line to the material. If material is not included in the article's Creative Commons licence and your intended use is not permitted by statutory regulation or exceeds the permitted use, you will need to obtain permission directly from the copyright holder. To view a copy of this licence, visit http://creativecommons.org/licenses/by/4.0/.

(C) The Author(s) 2021 